\title{
On affine usages in signal-based communication *
}

\author{
Roberto M. Amadio Mehdi Dogguy \\ Université Paris 7, PPS, UMR-7126
}

December 1, 2018

\begin{abstract}
We describe a type system for a synchronous $\pi$-calculus formalising the notion of affine usage in signal-based communication. In particular, we identify a limited number of usages that preserve affinity and that can be composed. As a main application of the resulting system, we show that typable programs are deterministic.
\end{abstract}

\section{Introduction}

We are interested in synchronous systems. In these systems, there is a notion of instant (or phase, or pulse, or round) and at each instant each component of the system, a thread, performs some actions and synchronizes with all the other threads. One may say that all threads proceed at the same speed and it is in this specific sense that we will refer to synchrony in this work.

Signal-based communication is often used as the basic interaction mechanism in synchronous systems (see, e.g., [5, 6]). Signals play a role similar to channels in asynchronous systems. Indeed a signal can be shared among several threads that can either emit or receive on it. There are however some important differences to keep in mind. First, the emission of a signal can never block the execution of a thread. Second, a signal emitted at a given instant can be received an arbitrary number of times within the same instant while it disappears in the following ones. Third, several values can be emitted on a signal during the same instant. Fourth, there are two forms of reception. In the first one, during the instant, one of the values emitted on the signal at that instant (if any) is received (non-deterministically), in the second one, at the end of the instant, one collects all the values emitted on the signal during the instant. The first form of reception may block the execution of the thread during the instant while the second one is never blocking.

Our goal in this paper is to study the notion of affine usage in this context. In particular, we will formalise our ideas in the context of a synchronous $\pi$-calculus ( $S \pi$-calculus) introduced in [2]. We assume that the reader is familiar with the $\pi$-calculus and proceed to give a flavour of the language (the formal definition of the $S \pi$-calculus is recalled in section 2).

The syntax of the $S \pi$-calculus is similar to the one of the $\pi$-calculus, however there are some important semantic differences that we highlight in the following simple example. Assume $v_{1} \neq v_{2}$ are two distinct values and consider the following program in $S \pi$ :

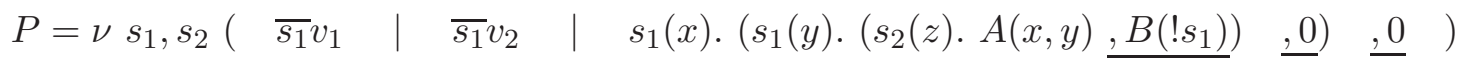

\footnotetext{
*Work partially supported by ANR-06-SETI-010-02.
} 
If we forget about the underlined parts and we regard $s_{1}, s_{2}$ as channel names then $P$ could also be viewed as a $\pi$-calculus process. In this case, $P$ would reduce to

$$
P_{1}=\nu s_{1}, s_{2}\left(s_{2}(z) \cdot A(\theta(x), \theta(y))\right.
$$

where $\theta$ is a substitution such that $\theta(x), \theta(y) \in\left\{v_{1}, v_{2}\right\}$ and $\theta(x) \neq \theta(y)$. In $S \pi$, signals persist within the instant and $P$ reduces to

$$
P_{2}=\nu s_{1}, s_{2}\left(\overline{s_{1}} v_{1}\left|\overline{s_{1}} v_{2}\right|\left(s_{2}(z) \cdot A(\theta(x), \theta(y)), \underline{B\left(! s_{1}\right)}\right)\right)
$$

where again $\theta(x), \theta(y) \in\left\{v_{1}, v_{2}\right\}$ but possibly $\theta(x)=\theta(y)$. What happens next? In the $\pi$ calculus, $P_{1}$ is deadlocked and no further computation is possible. In the $S \pi$-calculus, the fact that no further computation is possible in $P_{2}$ is detected and marks the end of the current instant. Then an additional computation represented by the relation $\stackrel{N}{\longrightarrow}$ moves $P_{2}$ to the following instant:

$$
P_{2} \stackrel{N}{\rightarrow} P_{2}^{\prime}=\nu s_{1}, s_{2} B(v)
$$

where $v \in\left\{\left[v_{1} ; v_{2}\right],\left[v_{2} ; v_{1}\right]\right\}$. Thus at the end of the instant, a dereferenced signal such as $! s_{1}$ becomes a list (possibly empty) of (distinct) values emitted on $s_{1}$ during the instant and then all signals are reset.

We continue our informal discussion with a few examples illustrating the kind of 'synchronous programming' that can be represented in the $S \pi$-calculus. The main point of these examples is to consider some critical issues in the determinacy of programs and to motivate the introduction of affine usages and set types (a kind of quotient types).

Example 1 (cell) We describe the behaviour of a generic cell that might be used in the simulation of a dynamic system. Each cell relies on three parameters: its state q, its own activation signal $s$, and the list $\ell$ of activation signals of its neighbours. The cell performs the following operations in a cyclic fashion: (i) it emits its current state along the activation signals of its neighbours, (ii) it waits till the end of the current instant (pause), and (iii) it collects the values emitted by its neighbours and computes its new state.

$$
\begin{array}{lll}
\operatorname{Cell}(q, s, \ell) & =\operatorname{Send}(q, s, \ell, \ell) & \\
\operatorname{Send}\left(q, s, \ell, \ell^{\prime}\right)=\left[\ell^{\prime} \unrhd \operatorname{cons}\left(s^{\prime}, \ell^{\prime \prime}\right)\right] & \left(\overline{s^{\prime}} q \mid \operatorname{Send}\left(q, s, \ell, \ell^{\prime \prime}\right)\right), \\
& \text { pause. } \operatorname{Cell}(\operatorname{next}(q, ! s), s, \ell)
\end{array}
$$

where next is a function that computes the following state of the cell according to its current state and the state of its neighbours. Assuming that the function next is invariant under permutations of the list of states, we would like to show that the evolution of the simulation is deterministic. To express this invariance, a natural idea is to treat the 'list' of distinct states as a 'set', i.e., as a list quotiented by a relation that identifies a list with any of its permutations.

Example 2 (synchronous data flow) We provide an example of synchronous data-flow computation. The network is described by the program

$$
\begin{aligned}
& \nu s_{2}, s_{3}, s_{4}, s_{5}\left(A\left(s_{1}, s_{2}, s_{3}, s_{4}\right)\left|B\left(s_{2}, s_{3}, s_{5}, s_{6}\right)\right| C\left(s_{4}, s_{5}\right)\right) \\
& \text { where: }\left\{\begin{array}{l}
A\left(s_{1}, s_{2}, s_{3}, s_{4}\right)=s_{1}(x) \cdot\left(\overline{s_{2}} f(x) \mid s_{3}(y) \cdot\left(\overline{s_{4}} g(y) \mid \text { pause. } A\left(s_{1}, s_{2}, s_{3}, s_{4}\right)\right), 0\right), 0 \\
\left.B\left(s_{2}, s_{3}, s_{5}, s_{6}\right)=s_{2}(x) \cdot\left(\overline{s_{3}} i(x)\left|s_{5}(y) \cdot\left(\overline{s_{6}} l(y)\right)\right| \text { pause. } B\left(s_{2}, s_{3}, s_{5}, s_{6}\right)\right), 0\right), 0 \\
C\left(s_{4}, s_{5}\right)
\end{array}\right.
\end{aligned}
$$


Assuming that at each instant at most one value is emitted on the input signal $s_{1}$, we would like to show that at each instant at most one value will be emitted on every other signal. This example suggests that we should introduce a notion of affine usage in signals.

The previous examples are really part of the folklore on synchronous programming (see, e.g., [11]). However, what is missing so far, is an analysis of what happens when we compose set types and affine usages as in the following example.

Example 3 (client-server) We describe first a 'server' handling a list of requests emitted in the previous instant on the signal s. For each request of the shape req $\left(s^{\prime}, x\right)$, it provides an answer which is a function of $x$ along the signal $s^{\prime}$.

$$
\begin{aligned}
& \operatorname{Server}(s)=\text { pause.Handle }(s, ! s) \\
& \operatorname{Handle}(s, \ell)=\left[\ell \unrhd \operatorname{cons}\left(\operatorname{req}\left(s^{\prime}, x\right), \ell^{\prime}\right)\right]\left(\overline{s^{\prime}} f(x) \mid \operatorname{Handle}\left(s, \ell^{\prime}\right)\right), \operatorname{Server}(s) .
\end{aligned}
$$

The programming of a client that issues a request $x$ on signal $s$ and returns the reply on signal $t$ could be the following:

$$
\operatorname{Client}(x, s, t)=\nu s^{\prime}\left(\bar{s} \text { req }\left(s^{\prime}, x\right) \mid \text { pause. } s^{\prime}(x) \cdot \bar{t} x, 0\right) .
$$

Let us first notice that a request contains a 'pointer', namely the name of the signal on which to answer the request. Then the 'folklore solution' of transforming a list of values into one value via an associative and commutative function does not work here. Indeed there seems to be no reasonable way to define an associative and commutative function on pointers. Instead, we look at Handle as a function from (a signal and) a list of requests to behaviours which is invariant under permutations of the list of requests. Note that to express this invariance we need a notion of behavioural equivalence and that this equivalence must satisfy the usual associativity and commutativity laws of parallel composition and must be preserved by parallel composition.

These considerations are enough to argue that the Server is a 'deterministic' program. No matter how many clients will issue requests at each instant, the Server will provide an answer to each of them in the following instant in a way which is independent of the order of the requests.

Let us now look at the Client. After issuing a request, the Client waits for a reply in the following instant. Clearly, if more than one reply comes, the outcome of the computation is not deterministic. For instance, we could have several 'Servers' running in parallel or a server could somehow duplicate the request. This means that the usage of the signal $s$ must be such that many 'clients' may issue a request but at most one 'server' may handle them at the end of the instant in an 'affine' way. Further, on the client side, the return signal s' can only be used to read while on the server side it can only be used to emit.

This preliminary discussion suggests the need for a formal analysis of the principles that allow to establish the determinacy of a synchronous program. This analysis will be obviously inspired by previous work on the foundations of linear logic [7], on linear typing of functional programs (e.g., [14]), and on linear usages of channels (e.g., [10]). Following this line of works, the analysis presented in section 3 will take the form of a typing system. The previous section 2, will recall the formal definition of the $S \pi$-calculus. In the final section 4, first we will introduce the properties of the typing system leading to a subject reduction theorem, and second we will describe a suitable notion of typed bisimulation and show that with respect to this notion, typable programs can be regarded as deterministic. 


\section{Definition of the $S \pi$-calculus}

We recall the formal definition of the $S \pi$-calculus and its bisimulation based semantics while referring the reader to [2, 4, for a deeper analysis. This section is rather technical but to understand the type system described in the following section 3 there are really just two points that the reader should keep in mind.

(1) The semantics of the calculus is given by the labelled transition system presented in table 2. A reader familiar with a $\pi$-calculus with asynchronous communication can understand these rules rather quickly. The main differences are: (1) the rule for emitting a signal formalises the fact that a signal, unlike a channel, persists within an instant and (2) the rules that describe the computation at the end of the instant.

(2) The labelled transition system induces a rather standard notion of bisimulation equivalence (definition (4) which is preserved by static contexts (fact 5) 1 In section 4, we will introduce a 'typed' definition of the bisimulation and show that with respect to this definition, typable programs are deterministic.

\section{$2.1 \quad$ Programs}

Programs $P, Q, \ldots$ in the $S \pi$-calculus are defined in table 1, We use the notation $\mathbf{m}$ for a vector $m_{1}, \ldots, m_{n}, n \geq 0$. The informal behaviour of programs follows. 0 is the terminated thread. $A(\mathbf{e})$ is a (tail) recursive call of a thread identifier $A$ with a vector $\mathbf{e}$ of expressions as argument; as usual the thread identifier $A$ is defined by a unique equation $A(\mathbf{x})=P$ such that the free variables of $P$ occur in $\mathbf{x}$. $\bar{s} e$ evaluates the expression $e$ and emits its value on the signal $s . s(x) . P, K$ is the present statement which is the fundamental operator of the model [1]. If the values $v_{1}, \ldots, v_{n}$ have been emitted on the signal $s$ then $s(x) . P, K$ evolves non-deterministically into $\left[v_{i} / x\right] P$ for some $v_{i}$ ([-/-] is our notation for substitution). On the other hand, if no value is emitted then the continuation $K$ is evaluated at the end of the instant. $\left[s_{1}=s_{2}\right] P_{1}, P_{2}$ is the usual matching function of the $\pi$-calculus that runs $P_{1}$ if $s_{1}$ equals $s_{2}$ and $P_{2}$, otherwise. Here both $s_{1}$ and $s_{2}$ are free. $[u \unrhd p] P_{1}, P_{2}$, matches $u$ against the pattern $p$. We assume $u$ is either a variable $x$ or a value $v$ and $p$ has the shape $\mathrm{c}(\mathbf{x})$, where $\mathrm{c}$ is a constructor and $\mathbf{x}$ is a vector of distinct variables. We also assume that if $u$ is a variable $x$ then $x$ does not occur free in $P_{1}$. At run time, $u$ is always a value and we run $\theta P_{1}$ if $\theta=\operatorname{match}(u, p)$ is the substitution matching $u$ against $p$, and $P_{2}$ if the substitution does not exist (written match $(u, p) \uparrow$ ). Note that as usual the variables occurring in the pattern $p$ (including signal names) are bound in $P_{1}$. $\nu s P$ creates a new signal name $s$ and runs $P$. $\left(P_{1} \mid P_{2}\right)$ runs in parallel $P_{1}$ and $P_{2}$. A continuation $K$ is simply a recursive call whose arguments are either expressions or values associated with signals at the end of the instant in a sense that we explain below. We will also write pause. $K$ for $\nu s s(x) .0, K$ with $s$ not free in $K$. This is the program that waits till the end of the instant and then evaluates $K$.

\subsection{Expressions}

Expressions are partitioned in several syntactic categories as specified in table 1. As in the $\pi$-calculus, signal names stand both for signal constants as generated by the $\nu$ operator and signal variables as in the formal parameter of the present operator. Variables Var include

\footnotetext{
${ }^{1}$ As a matter of fact the labelled transition system is built so that the definition of bisimulation equivalence looks standard [4].
} 


\begin{tabular}{|c|c|c|}
\hline$P$ & $::=0\|A(\mathbf{e})\| \bar{s} e\|s(x) . P, K\|$ & (programs) \\
\hline$K$ & $\begin{aligned} & {\left[s_{1}=s_{2}\right] P_{1}, P_{2}\left\|[u \unrhd p] P_{1}, P_{2}\left|\nu s P \| P_{1}\right| P_{2}\right.} \\
::= & A(\mathbf{r})\end{aligned}$ & (continuation next instant) \\
\hline Sig & $::=s\|t\| \cdots$ & (signal names) \\
\hline $\operatorname{Var}$ & $:=\operatorname{Sig}\|x\| y\|z\| \cdots$ & (variables) \\
\hline Cnst & $::=* \|$ nil $\|$ cons $\|\mathrm{c}\| \mathrm{d} \| \cdots$ & (constructors) \\
\hline Val & $::=\operatorname{Sig} \| \operatorname{Cnst}(\mathrm{Val}, \ldots, \mathrm{Val})$ & (values $v, v^{\prime}, \ldots$ ) \\
\hline Pat & $::=\operatorname{Cnst}(\operatorname{Var}, \ldots, \operatorname{Var})$ & (patterns $p, p^{\prime}, \ldots$ ) \\
\hline Fun & $::=f\|g\| \cdots$ & (first-order function symbols) \\
\hline Exp & $::=\operatorname{Var}\|\operatorname{Cnst}(\operatorname{Exp}, \ldots, \operatorname{Exp})\| \operatorname{Fun}(\operatorname{Exp}, \ldots, \operatorname{Exp})$ & $\left(\right.$ expressions $\left.e, e^{\prime}, \ldots\right)$ \\
\hline $\operatorname{Rexp}$ & $\begin{aligned}::= & ! \operatorname{Sig}\|\operatorname{Var}\| \operatorname{Cnst}(\operatorname{Rexp}, \ldots, \operatorname{Rexp}) \| \\
& \quad \operatorname{Fun}(\operatorname{Rexp}, \ldots, \operatorname{Rexp})\end{aligned}$ & (exp. with deref. $\left.r, r^{\prime}, \ldots\right)$ \\
\hline
\end{tabular}

Table 1: Syntax of programs and expressions

signal names as well as variables of other types. Constructors Cnst include $*$, nil, and cons. Values $\mathrm{Val}$ are terms built out of constructors and signal names. Patterns Pat are terms built out of constructors and variables (including signal names). If $P, p$ are a program and a pattern then we denote with $f n(P), f n(p)$ the set of free signal names occurring in them, respectively. We also use $F V(P), F V(p)$ to denote the set of free variables (including signal names). We assume first-order function symbols $f, g, \ldots$ and an evaluation relation $\Downarrow$ such that for every function symbol $f$ and values $v_{1}, \ldots, v_{n}$ of suitable type there is a unique value $v$ such that $f\left(v_{1}, \ldots, v_{n}\right) \Downarrow v$ and $f n(v) \subseteq \bigcup_{i=1, \ldots, n} f n\left(v_{i}\right)$. Expressions Exp are terms built out of variables, constructors, and function symbols. The evaluation relation $\Downarrow$ is extended in a standard way to expressions whose only free variables are signal names. Finally, Rexp are expressions that may include the value associated with a signal $s$ at the end of the instant (which is written ! $s$, following the ML notation for dereferenciation). Intuitively, this value is a list of values representing the set of values emitted on the signal during the instant.

The definition of a simple type system for the $S \pi$-calculus can be extracted from the more elaborate type system presented in section 3 by confusing 'set-types' with 'list-types' and by neglecting all considerations on usages.

\subsection{Actions}

The syntactic category act of actions described in table 2 comprises relevant, auxiliary, and nested actions. The relevant actions are those that are actually considered in the bisimulation game. They consist of: (i) an internal action $\tau$, (ii) an emission action $\nu \mathbf{t} \bar{s} v$ where it is assumed that the signal names $\mathbf{t}$ are distinct, occur in $v$, and differ from $s$, (iii) an input action $s v$, and (iv) an action $N$ (for Next) that marks the move from the current to the next instant.

The auxiliary actions consist of an input action $s ? v$ which is coupled with an emission action in order to compute a $\tau$ action and an action $(E, V)$ which is just needed to compute an action $N$. The latter is an action that can occur exactly when the program cannot perform $\tau$ actions and it amounts to (i) collect in lists the set of values emitted on every signal, (ii) to reset all signals, and (iii) to initialise the continuation $K$ for each present statement of the shape $s(x) . P, K$.

In order to formalise these three steps we need to introduce some notation. Let $E$ vary over functions from signal names to finite sets of values. Denote with $\emptyset$ the function that 
associates the empty set with every signal name, with $[M / s]$ the function that associates the set $M$ with the signal name $s$ and the empty set with all the other signal names, and with $\cup$ the union of functions defined point-wise.

We represent a set of values as a list of the values belonging to the set. More precisely, we write $v \|-M$ and say that $v$ represents $M$ if $M=\left\{v_{1}, \ldots, v_{n}\right\}$ and $v=\left[v_{\pi(1)} ; \ldots ; v_{\pi(n)}\right]$ for some permutation $\pi$ over $\{1, \ldots, n\}$. Suppose $V$ is a function from signal names to lists of values. We write $V \|-E$ if $V(s) \|-E(s)$ for every signal name $s$. We also write $\operatorname{dom}(V)$ for $\{s \mid V(s) \neq[]\}$. If $K$ is a continuation, i.e., a recursive call $A(\mathbf{r})$, then $V(K)$ is obtained from $K$ by replacing each occurrence ! $s$ of a dereferenced signal with the associated value $V(s)$. We denote with $V[\ell / s]$ the function that behaves as $V$ except on $s$ where $V[\ell / s](s)=\ell$.

With these conventions, a transition $P \stackrel{(E, V)}{\longrightarrow} P^{\prime}$ intuitively means that $(1) P$ is suspended, (2) $P$ emits exactly the values specified by $E$, and (3) the behaviour of $P$ in the following instant is $P^{\prime}$ and depends on $V$. It is convenient to compute these transitions on programs where all name generations are lifted at top level. We write $P \succeq Q$ if we can obtain $Q$ from $P$ by repeatedly transforming, for instance, a subprogram $\nu s P^{\prime} \mid P^{\prime \prime}$ into $\nu s\left(P^{\prime} \mid P^{\prime \prime}\right)$ where $s \notin f n\left(P^{\prime \prime}\right)$.

Finally, the nested actions $\mu, \mu^{\prime}, \ldots$ are certain actions (either relevant or auxiliary) that can be produced by a sub-program and that we need to propagate to the top level.

\subsection{Labelled transition system and bisimulation}

The labelled transition system is defined in table 2 where rules apply to programs whose only free variables are signal names and with standard conventions on the renaming of bound names. As usual, one can rename bound variables, and symmetric rules are omitted. The first 12 rules from (out) to $\left(\nu_{e x}\right)$ are quite close to those of a polyadic $\pi$-calculus with asynchronous communication (see [8, 3]) with the following exception: rule (out) models the fact that the emission of a value on a signal persists within the instant. The last 5 rules from (0) to (next) are quite specific of the $S \pi$-calculus and determine how the computation is carried on at the end of the instant (cf. discussion in 2.3).

We derive from the labelled transition system a notion of (weak) labelled bisimulation. First define $\stackrel{\alpha}{\Rightarrow}$ as $(\stackrel{\tau}{\rightarrow})^{*}$ if $\alpha=\tau,(\stackrel{\tau}{\Rightarrow}) \circ(\stackrel{N}{\rightarrow})$ if $\alpha=N$, and $(\stackrel{\tau}{\Rightarrow}) \circ(\stackrel{\alpha}{\rightarrow}) \circ(\stackrel{\tau}{\Rightarrow})$ otherwise. This is the standard definition except that we insist on not having internal reductions after an $N$ action. Intuitively, we assume that an observer can control the execution of programs so as to be able to test them at the very beginning of each instant. We write $P \stackrel{\alpha}{\rightarrow}$. for $\exists P^{\prime}\left(P \stackrel{\alpha}{\rightarrow} P^{\prime}\right)$.

Definition 4 (labelled bisimulation) A symmetric relation $\mathcal{R}$ on programs is a labelled bisimulation if $P \mathcal{R} Q, P \stackrel{\alpha}{\rightarrow} P^{\prime}$, bn $(\alpha) \cap f n(Q)=\emptyset$ implies $\exists Q^{\prime}\left(Q \stackrel{\alpha}{\Rightarrow} Q^{\prime}, \quad P^{\prime} \mathcal{R} Q^{\prime}\right)$. We denote with $\approx$ the largest labelled bisimulation.

Fact 5 ([4]) Labelled bisimulation is preserved by parallel composition and name generation.

\section{$3 \quad$ An affine type system}

An analysis of the notion of determinacy carried on in [4], along the lines of [12], suggests that there are basically two situations that need to be analysed in order to guarantee the determinacy of programs. (1) At least two distinct values compete to be received within an 


$$
\begin{aligned}
& \begin{array}{|lll|}
\hline \text { act } & ::=\alpha \| \text { aux } & \text { (actions) } \\
\alpha & ::=\tau\|\nu \mathrm{t} \bar{s} v s v\| N & \text { (relevant actions) } \\
\text { aux } & ::=s ? v \|(E, V) & \text { (auxiliary actions) } \\
\mu & ::=\tau \| \nu \mathrm{t} \bar{s} v \mid s ? v & \text { (nested actions) } \\
\hline
\end{array} \\
& \text { (out) } \frac{e \Downarrow v}{\bar{s} e \stackrel{\overline{s v}}{\longrightarrow} \bar{s} e} \quad\left(i n_{\text {aux }}\right) \frac{}{s(x) \cdot P, K \stackrel{s ? v}{\longrightarrow}[v / x] P}
\end{aligned}
$$

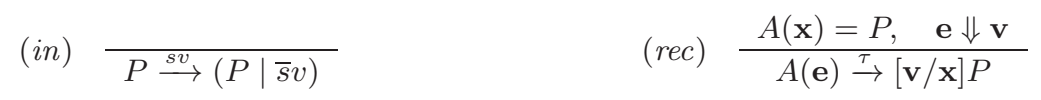

$$
\begin{aligned}
& \left(={ }_{1}^{s i g}\right) \frac{s_{1} \neq s_{2}}{[s=s] P_{1}, P_{2} \stackrel{\tau}{\rightarrow} P_{1}} \quad\left(=_{2}^{s i g}\right) \quad \frac{\tau}{\left[s_{1}=s_{2}\right] P_{1}, P_{2} \stackrel{\tau}{\rightarrow} P_{2}} \\
& \left(={ }_{1}^{\text {ind }}\right) \quad \frac{\operatorname{match}(v, p)=\theta}{[v \unrhd p] P_{1}, P_{2} \stackrel{\tau}{\rightarrow} \theta P_{1}} \quad\left(=_{1}^{\text {ind }}\right) \quad \frac{\operatorname{match}(v, p)=\uparrow}{[v \unrhd p] P_{1}, P_{2} \stackrel{\tau}{\rightarrow} P_{2}}
\end{aligned}
$$

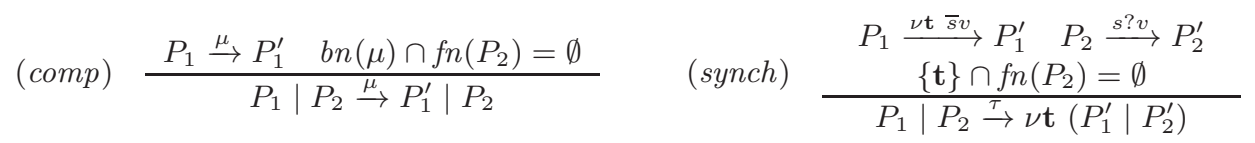

$$
\begin{aligned}
& (\nu) \frac{P \stackrel{\mu}{\rightarrow} P^{\prime} t \notin n(\mu)}{\nu t P \stackrel{\mu}{\rightarrow} \nu t P^{\prime}} \quad\left(\nu_{e x}\right) \frac{P \stackrel{\nu \mathbf{t} \bar{s} v}{\longrightarrow} P^{\prime} \quad t^{\prime} \neq s \quad t^{\prime} \in n(v) \backslash\{\mathbf{t}\}}{\nu t^{\prime} P \stackrel{\left(\nu t^{\prime}, \mathbf{t}\right) \bar{s} v}{\longrightarrow} P^{\prime}}
\end{aligned}
$$

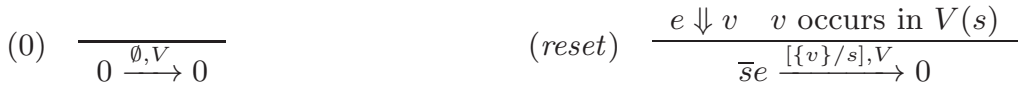

$$
\begin{aligned}
& \text { (cont) } \frac{s \notin \operatorname{dom}(V)}{s(x) \cdot P, K \stackrel{\emptyset, V}{\longrightarrow} V(K)} \quad(\text { par }) \quad \frac{P_{i} \stackrel{E_{i}, V}{\longrightarrow} P_{i}^{\prime}}{\left(P_{1} \mid P_{2}\right) \stackrel{E_{1} \cup E_{2}, V}{\longrightarrow}\left(P_{1}^{\prime} \mid P_{2}^{\prime}\right)} \\
& \text { (next) } \frac{P \succeq \nu \mathbf{s} P^{\prime} \quad P^{\prime} \stackrel{E, V}{\longrightarrow} P^{\prime \prime} \quad V \|-E}{P \stackrel{N}{\longrightarrow} \nu \mathbf{s} P^{\prime \prime}}
\end{aligned}
$$

Table 2: Labelled transition system 
instant, for instance, consider: $\bar{s} v_{1}\left|\bar{s} v_{2}\right| s(x) . P, K$. (2) At the end of the instant, at least two distinct values are available on a signal. For instance, consider: $\bar{s} v_{1}\left|\bar{s} v_{2}\right|$ pause. $A(! s)$. A sensible approach is to avoid completely the first situation and to allow the second provided the behaviour of the continuation $A$ does not depend on the order in which the values are collected. Technically, we consider a notion of affine signal usage to guarantee the first condition and a notion of set type for the second one. While this is a good starting point, it falls short of providing a completely satisfying answer because the type constructions do not compose very well. Then our goal is to discover a collection of signal usages with better compositionality properties. The outcome of our analysis are three main additional usages which are described below as $e_{3-5}$ in table 3 (left part).

\subsection{Usages}

In first approximation, we may regard a usage as an element of the set $L=\{0,1, \infty\}$ with the intuition that 0 corresponds to no usage at all, 1 to at most one usage, and $\infty$ to any usage. We add usages with a partial operation $\oplus$ such that $0 \oplus x=x \oplus 0=x$ and $\infty \oplus \infty=\infty$, and which is undefined otherwise (note in particular that $1 \oplus 1$ is undefined). The addition induces an order by $x \leq y$ if $\exists z x \oplus z=y$. With respect to this order, 0 is the least element while 1 and $\infty$ are incomparable. If $x \geq y$ then we define a subtraction operation $x \ominus y$ as the largest $z$ such that $x=y \oplus z$. Therefore: $x \ominus 0=x, 1 \ominus 1=0$, and $\infty \ominus \infty=\infty$.

This classification of usages is adequate when handling purely functional data where the intuition is that data with usage 1 have at most one reference to them. However, when handling more complex entities such as references, channels, or signals it is convenient to take a more refined view. Specifically, a signal usage can be refined to include information about whether a signal is used: (i) to emit, (ii) to receive during the instant, or (iii) to receive at the end of the instant. Then a usage becomes an element of $L^{3}$.

Among the 27 possible usages of the shape $(x, y, z)$ for $x, y, z \in\{0,1, \infty\}$, we argue that there are 5 main ones as described in table 3 (left part). First of all, we must have $x \neq 0$ and $(y \neq 0 \vee z \neq 0)$ since a signal on which we cannot send or receive has no interest. Now if $x=\infty$ then we are forced to take $y=0$ since we want to preserve the determinacy. Then for $z=\infty$ we have the usage $e_{1}$ and for $z=1$ we have the usage $e_{3}$. Suppose now $x=1$. One choice is to have $y=z=\infty$ and then we have the usage $e_{2}$. On the other hand if we want to preserve affinity then we should receive the emitted value at most once. Hence we have $y=0, z=1$ or $y=1, z=0$ which correspond to the usages $e_{4}$ and $e_{5}$, respectively. From these 5 main usages within an instant, we obtain the derived ones (see again table 3 ) by simply turning one or more 1's to 0 's.

In a synchronous framework, it makes sense to consider how usages vary over time. The simplest solution would be to look at usages of the shape $x^{\omega}$ which are invariant under time. However, to reason effectively on the programs, we are led to consider usages of the shape $x y^{\omega}$ where $x, y \in L^{3}$ are two usages of the same kind $(1-5)$. Overall, we have 5 kinds of usages: kind 1 contains 1 usage, kinds 2 and 3 contain 4 usages each, and kinds 4 and 5 contain 16 usages each. The fact that $(1,0,0)$ and $(0,0,0)$ can occur both in the usages of kind 4 and 5 is a slight source of ambiguity. Whenever needed, this ambiguity can be resolved by assuming that the kind of the usage is made explicit. We denote with $U$ the set of all these $1+4+4+16+16$ usages and with $U(i)$ the set of usages of kind $i$, for $i=1, \ldots, 5$. We consider that the addition operation $\oplus$ is defined only if $u, u^{\prime} \in U(i)$ and $u \oplus u^{\prime} \in U(i)$ for some $i \in\{1, \ldots, 5\}$. If $u \in U$ then $\uparrow u$, the shift of $u$, is the infinite word in $U$ obtained 


\begin{tabular}{l|l||c|c|c|c} 
main usages & derived usages & $x y^{\omega} \in U(i)$ is & affine & uniform & aff. preserving \\
\hline$e_{1}=(\infty, 0, \infty)$ & - & $i=1$ & no & yes & no \\
$e_{2}=(1, \infty, \infty)$ & $(0, \infty, \infty)$ & $i=2$ & yes $/$ no & yes $/$ no & no \\
$e_{3}=(\infty, 0,1)$ & $(\infty, 0,0)$ & $i=3$ & yes $/$ no & yes $/$ no & yes \\
$e_{4}=(1,0,1)$ & $(1,0,0),(0,0,1),(0,0,0)$ & $i=4$ & yes $/$ no & yes $/$ no & yes \\
$e_{5}=(1,1,0)$ & $(1,0,0),(0,1,0),(0,0,0)$ & $i=5$ & yes $/$ no & yes $/$ no & yes
\end{tabular}

Table 3: Usages and their classification

from $u$ by removing the first character. This operation is always defined. If $u$ is a usage (an infinite word), then $u(i)$ for $i \geq 0$ denotes its $i^{t h}$ character and $u(i)_{j}$ for $j \in\{1,2,3\}$ the $j^{\text {th }}$ component of $u(i)$.

We classify the usages according to 3 properties: affinity, uniformity, and preservation of affinity. We say that a usage is affine if it contains a 1 and non-affine otherwise. We also say that it is uniform if it is of the shape $x^{\omega}$ and that it is neutral if it is the neutral element with respect to the addition $\oplus$ on the set of usages $U(i)$ to which it belong. It turns out that the non-affine usages are always uniform and moreover they coincide with the neutral ones. Finally, by definition, the usages in the sets $U(i)$ for $i=3,4,5$ are affine preserving The classification is summarised in the table 3 (right part).

\subsection{Types}

In first approximation, types are either inductive types or signal types. As usual, an inductive type such as the type $\operatorname{List}(\sigma)$ of lists of elements of type $\sigma$ is defined by an equation $\operatorname{List}(\sigma)=$ nil $\|$ cons of $\sigma, \operatorname{List}(\sigma)$ specifying the ways in which an element of this type can be built.

In our context, inductive types come with a usage $x$ which belongs to the set $\{1, \infty\}$ and which intuitively specifies whether the values of this type can be used at most once or arbitrarily many times (once more we recall that 1 and $\infty$ are incomparable). To summarise, if $\sigma_{1}, \ldots, \sigma_{k}$ are types already defined then an inductive type $C_{x}\left(\sigma_{1}, \ldots, \sigma_{k}\right)$ is specified by an equation $C_{x}\left(\sigma_{1}, \ldots, \sigma_{n}\right)=\cdots \mid$ c of $\sigma_{1}^{\prime}, \ldots, \sigma_{m}^{\prime} \cdots$ where the types $\sigma_{j}^{\prime}, j=1, \ldots, m$ are either one of the types $\sigma_{i}, i=1, \ldots, n$ or the inductive type $C_{x}(\ldots)$ being defined. There is a further constraint that has to be respected, namely that if one of the types $\sigma_{i}$ is 'affine' then the usage $x$ must be affine preserving, i.e., $x=1$. An affine type is simply a type which contains an affine usage. The following grammar will provide a precise definition of the affine types.

When collecting the values at the end of the instant we will also need to consider set types. They are described by an equation $\operatorname{Set}_{x}(\sigma)=$ nil $\|$ cons of $\sigma, \operatorname{Set}_{x}(\sigma)$ which is quite similar to the one for lists. Note that set types too come with a usage $x \in\{1, \infty\}$ and that if $\sigma$ is an affine type then the usage $x$ must be affine preserving. The reader might have noticed that we take the freedom of using the constructor nil both with the types $\operatorname{List}_{u}(\sigma)$ and $\operatorname{Set}_{u}(\sigma)$, $u \in\{1, \infty\}$, and the constructor cons both with the types $\left(\sigma, \operatorname{List}_{u}(\sigma)\right) \rightarrow \operatorname{List}_{u}(\sigma)$ and $\left(\sigma, \operatorname{Set}_{u}(\sigma)\right) \rightarrow \operatorname{Set}_{u}(\sigma)$. However, one should assume that a suitable label on the constructors will allow to disambiguate the situation.

Finally, we denote with $\operatorname{Sig}_{u}(\sigma)$ the type of signals carrying values of type $\sigma$ according to the usage $u$. As for inductive and set types, if $\sigma$ is an affine type then the usage $u$ must be affine preserving. As we have seen, the collection of usages for signals is much richer than for inductive data types. We require that the non-uniform (i.e. type-dependent) usages can only 
appear at top level.

To formalise these distinctions, we are lead to use several names for types as specified in table 4. We denote with $\kappa$ non-affine (or classical) types, i.e., types that carry no affine usage information. These types have a uniform usage. We denote with $\lambda$ affine and uniform types. The types $\sigma, \sigma^{\prime}, \ldots$ stand for types with uniform usage (either non-affine or affine). Finally, the types $\rho, \rho^{\prime}, \ldots$ include all the previous one plus types that have a non-uniform usage. We notice that classical uniform types can be nested in an arbitrary way, while affine uniform types can only be nested under type constructors that preserve affinity. Moreover, types with non-uniform usages (either classical or affine) cannot be nested at all.

The partial operation of addition $\oplus$ is extended to types so that: $O p_{u_{1}}(\sigma) \oplus O p_{u_{2}}(\sigma)=$ $O p_{u_{1} \oplus u_{2}}(\sigma)$, where $O p$ can be $C$, Set, or Sig, and provided that $u_{1} \oplus u_{2}$ is defined. For instance, $\operatorname{List}_{1}(\lambda) \oplus \operatorname{List}_{1}(\lambda)$ is undefined because $1 \oplus 1$ is not defined.

A type context (or simply a context) $\Gamma$ is a partial function with finite domain $\operatorname{dom}(\Gamma)$ from variables to types. An addition operation $\Gamma_{1} \oplus \Gamma_{2}$ on contexts is defined if and only if for all $x$ such that $\Gamma_{1}(x)=\rho_{1}$ and $\Gamma_{2}(x)=\rho_{2}$, the type $\rho_{1} \oplus \rho_{2}$ is defined. The shift operation is extended to contexts so that $(\uparrow \Gamma)(x)=\operatorname{Sig}_{(\uparrow u)}(\sigma)$ if $\Gamma(x)=\operatorname{Sig}_{u}(\sigma)$ and $(\uparrow \Gamma)(x)=\Gamma(x)$ otherwise. We also denote with $\Gamma, x: \sigma$ the context $\Gamma$ extended with the pair $x: \sigma$ (so $x \notin \operatorname{dom}(\Gamma)$ ). We say that a context is neutral (uniform) if it assigns to variables neutral (uniform) types.

\subsection{Semantic instrumentation}

As we have seen, each signal belongs to exactly one of 5 kinds of usages. Let us consider in particular the kind 5 whose main usage is $u_{5}=e_{5}^{\omega}$. The forthcoming type system is supposed to guarantee that a value emitted on a signal of kind 5 is received at most once during an instant. Now, suppose we consider the program $\bar{s} t \mid s(x) . \bar{x}, 0$ and we attribute a usage $u_{5}$ to the signals $s$ and $t$. According to this usage this program should be well typed. However, if we apply the labelled transition system in table 2, this program reduces to $(\bar{s} t \mid \bar{t})$ which fails to be well-typed because the double occurrence of $t$ is not compatible with an affine usage of $t$. Intuitively, after the signal $s$ has been read once no other synchronisation should arise during the instant either within the program or with the environment. To express this fact we proceed as follows. First, we instrument the semantics so that it marks (underlines) the emissions on signals of kind 5 that have been used at least once during the instant. The emission has no effect on the labelled transition system in the sense that $\underline{\bar{s} e}$ behaves exactly as $\bar{s} e$.

$$
\text { (out) } \frac{e \Downarrow v}{\bar{s} e \stackrel{\bar{s} v}{\longrightarrow} \underline{\bar{s} e}} \quad(\underline{\text { out }}) \frac{e \Downarrow v}{\underline{\bar{s} e} \stackrel{\bar{s} v}{\longrightarrow} \underline{\bar{s} e}} \quad(\underline{\text { reset }}) \quad \frac{e \Downarrow v \quad v \text { occurs in } V(s)}{\underline{\bar{s} e} \stackrel{[\{v\} / s], V}{\longrightarrow} 0}
$$

On the other hand, we introduce a special rule $(\underline{o u t})$ to type $\underline{\bar{s} e}$ which requires at least a usage $(1,1,0) \cdot(0,0,0)^{\omega}$ for the signal $s$ while neglecting the expression $e$. By doing this, we make sure that a second attempt to receive on $s$ will produce a type error. In other terms, if typing is preserved by 'compatible' transitions, then we can be sure that a value emitted on a signal of kind 5 is received at most once within an instant.

\subsection{Type system}

The type system is built around few basic ideas. 
(1) Usages including both input and output capabilities can be decomposed in simpler ones. For instance, $(1,1,0)^{\omega}=(1,0,0)(0,1,0)^{\omega} \oplus(0,1,0)(1,0,0)^{\omega}$.

(2) A rely-guarantee kind of reasoning: when we emit a value we guarantee certain resources while when we receive a value we rely on certain resources.

(3) Every affine usage can be consumed at most once in the typing judgement (and in the computation).

When formalising the typing judgements we need to distinguish the typing of an expression $e$ from the typing of an expression with dereferenciation $r$ and the typing of a recursive call $A\left(e_{1}, \ldots, e_{n}\right)$ from the typing of a recursive call at the end of the instant $A\left(r_{1}, \ldots, r_{n}\right)$. To do this we will write $[r]$ rather than $r$ and $\left[A\left(r_{1}, \ldots, r_{n}\right)\right]$ rather than $A\left(r_{1}, \ldots, r_{n}\right)$.

We will consider four typing judgements: $\Gamma \vdash e: \rho, \Gamma \vdash[r]: \rho, \Gamma \vdash P$, and $\Gamma \vdash$ $\left[A\left(r_{1}, \ldots, r_{n}\right)\right]$, and we wish to refer to them with a uniform notation $\Gamma \vdash U: T$. To this end, we introduce a fictious type $P r$ of programs and regard the judgements $\Gamma \vdash P: P r$ and $\Gamma \vdash\left[A\left(r_{1}, \ldots, r_{n}\right)\right]: P r$ as an expansion of $\Gamma \vdash P$ and $\Gamma \vdash\left[A\left(r_{1}, \ldots, r_{n}\right)\right]$, respectively. Then we let $U$ stand for one of $e,[r], P,\left[A\left(r_{1}, \ldots, r_{n}\right)\right]$, and $T$ for one of $\rho, \operatorname{Pr}$.

We assume that function symbols are given non-affine types of the shape $\left(\kappa_{1}, \ldots, \kappa_{n}\right) \rightarrow \kappa$. We denote with $k$ either a constructor or a function symbol and we assume that its type is explicitly given.

The typing rules are given in table 4. We comment first the typing rules for the expressions. We notice that the arguments and the result of a constructor or a function symbol have always a uniform type. The rules $\left(!_{S e t}\right)$ and $\left(!_{\text {List }}\right)$ describe the type of a dereferenced signal following its usage. If the usage is of kind 1 then the list of values associated with the signal at the end of the instant must be treated as a set, if the usage is of kind 2 then we know that the list of values contains at most one element and therefore its processing will certainly be 'order-independent', if the usage is of kind 3 then the list may contain several values and it must be processed as an affine set, finally if the usage is of kind 4 (the usage of kind 5 forbids reception at the end of the instant) then again the list of values will contain at most one element so we can rely on an affine list type.

Notice the special form of the rule $\left[v a r_{s i g}\right]$. The point here is that in a recursive call $K=A(! s, s)$ at the end of instant, we need to distinguish the resources needed to type ! $s$ which should relate to the current instant from the resources needed to type $s$ which should relate to the following instants. For instance, we want to type $K$ in a context $s: \operatorname{Sig}_{u}(\sigma)$ where $u=(0,0,1)^{\omega}$. This is possible because we can decompose $u$ in $u_{1} \oplus u_{2}$, where $u_{1}=$ $(0,0,1)(0,0,0)^{\omega}$ and $u_{2}=(0,0,0)(0,0,1)^{\omega}$, and we can rely on $u_{1}$ to type [!s] and on $u_{2}$ to type $[s]$ (by $\left[\right.$ var $\left._{\text {sig }}\right]$ ).

A set-type is a particular case of quotient type and therefore its definition goes through the definition of an equivalence relation $\sim_{\rho}$ on values. This is defined as the least equivalence relation such that $s \sim_{\operatorname{Sig}_{u}(\sigma)} s, \mathrm{C} \sim_{C(\sigma)} \mathrm{C}$, if $\mathrm{c}$ is a constant of type $C(\sigma)$, and

$$
\begin{array}{ll}
\mathrm{c}\left(v_{1}, \ldots, v_{n}\right) \sim_{C_{u}\left(\sigma_{1}, \ldots, \sigma_{n}\right)} \mathrm{c}\left(u_{1}, \ldots, u_{n}\right) & \text { if } v_{i} \sim_{\sigma_{i}} u_{i} \text { for } i=1, \ldots, n \\
{\left[v_{1} ; \ldots ; v_{n}\right] \sim_{\operatorname{Set}_{u}(\sigma)}\left[u_{1} ; \ldots ; u_{m}\right]} & \text { if }\left\{v_{1}, \ldots, v_{n}\right\} \sim_{\operatorname{Set}_{u}(\sigma)}\left\{u_{1}, \ldots, u_{m}\right\}, \\
\text { where: }\left\{v_{1}, \ldots, v_{n}\right\} \sim_{\operatorname{Set}_{u}(\sigma)}\left\{u_{1}, \ldots, u_{m}\right\} & \text { if for a permutation } \pi, v_{i} \sim_{\sigma} u_{\pi(i)} .
\end{array}
$$

Furthermore, we assume that each function symbol $f$, coming with a (classical) type $\left(\kappa_{1}, \ldots, \kappa_{n}\right) \rightarrow \kappa$, respects the typing in the following sense: (1) if $v_{i} \sim_{\kappa_{i}} u_{i}, i=1, \ldots, n$, $f\left(v_{1}, \ldots, v_{n}\right) \Downarrow v$ and $f\left(u_{1}, \ldots, u_{n}\right) \Downarrow u$ then $v \sim_{\kappa} u$. (2) If $\Gamma \vdash f\left(v_{1}, \ldots, v_{n}\right): \kappa$ and $f\left(v_{1}, \ldots, v_{n}\right) \Downarrow v$ then $\Gamma \vdash v: \kappa$. 


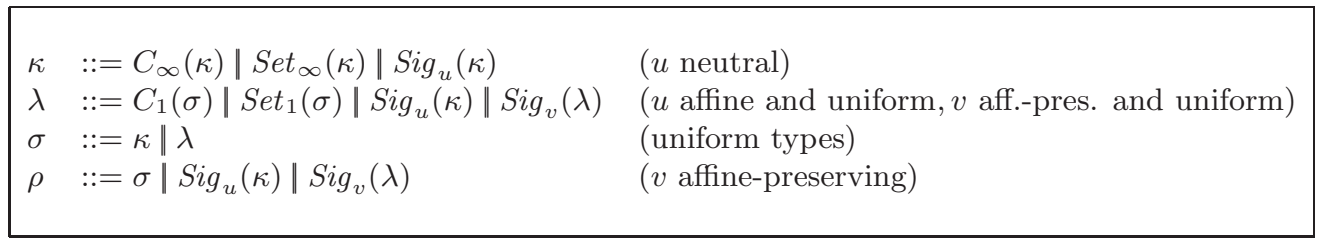

$$
\begin{aligned}
& (\text { var }) \frac{u \geq u^{\prime} \quad O p \in\{\text { Sig, Set }, C\}}{\Gamma, x: O p_{u}(\sigma) \vdash x: O p_{u^{\prime}}(\sigma)} \quad(k) \begin{array}{c}
\Gamma_{i} \vdash e_{i}: \sigma_{i} \quad i=1, \ldots, n \\
k:\left(\sigma_{1}, \ldots, \sigma_{n}\right) \rightarrow \sigma \quad k=f \text { or } k=\mathrm{c} \\
\hline \Gamma_{0} \oplus \Gamma_{1} \oplus \cdots \oplus \Gamma_{n} \vdash k\left(e_{1}, \ldots, e_{n}\right): \sigma
\end{array} \\
& {\left[\operatorname{var}_{C}\right] \frac{O p=C \quad O p=\operatorname{Set}}{\Gamma, x: O p_{u}(\sigma) \vdash[x]: O p_{u}(\sigma)} \quad\left[\operatorname{var}_{s i g}\right] \quad \frac{y^{\omega} \geq u}{\Gamma, s: \operatorname{Sig}_{x y^{\omega}}(\sigma) \vdash[s]: \operatorname{Sig}_{u}(\sigma)}} \\
& \Gamma_{i} \vdash\left[r_{i}\right]: \sigma_{i} \quad i=1, \ldots, n \\
& {[k] \frac{k:\left(\sigma_{1}, \ldots, \sigma_{n}\right) \rightarrow \sigma \quad k=f \text { or } k=\mathrm{c}}{\Gamma_{0} \oplus \Gamma_{1} \oplus \cdots \oplus \Gamma_{n} \vdash\left[k\left(r_{1}, \ldots, r_{n}\right)\right]: \sigma}} \\
& (u(0) \geq(\infty, 0, \infty) \wedge x=\infty) \vee \\
& {\left[!_{\text {Set }}\right] \quad(u(0) \geq(\infty, 0,1) \wedge x=1)} \\
& \Gamma, s: \operatorname{Sig}_{u}(\sigma) \vdash[! s]: \operatorname{Set}_{x}(\sigma) \\
& \text { (0) } \overline{\Gamma \vdash 0} \\
& (\nu) \frac{\Gamma, s: \operatorname{Sig}_{u}(\sigma) \vdash P}{\Gamma \vdash \nu s: \operatorname{Sig}_{u}(\sigma) P} \\
& \begin{array}{c}
s_{1}, s_{2} \in \operatorname{dom}(\Gamma) \\
\left(m_{s}\right) \quad \frac{\Gamma \vdash P_{i} \quad i=1,2}{\Gamma \vdash\left[s_{1}=s_{2}\right] P_{1}, P_{2}}
\end{array} \\
& \text { (par) } \frac{\Gamma_{i} \vdash P_{i} \quad i=1,2}{\Gamma_{1} \oplus \Gamma_{2} \vdash P_{1} \mid P_{2}} \\
& \text { (out) } \frac{\Gamma \vdash s: \operatorname{Sig}_{u}(\sigma) u(0)=(1,1,0)}{\Gamma \vdash \underline{\bar{s}} \underline{ }}
\end{aligned}
$$

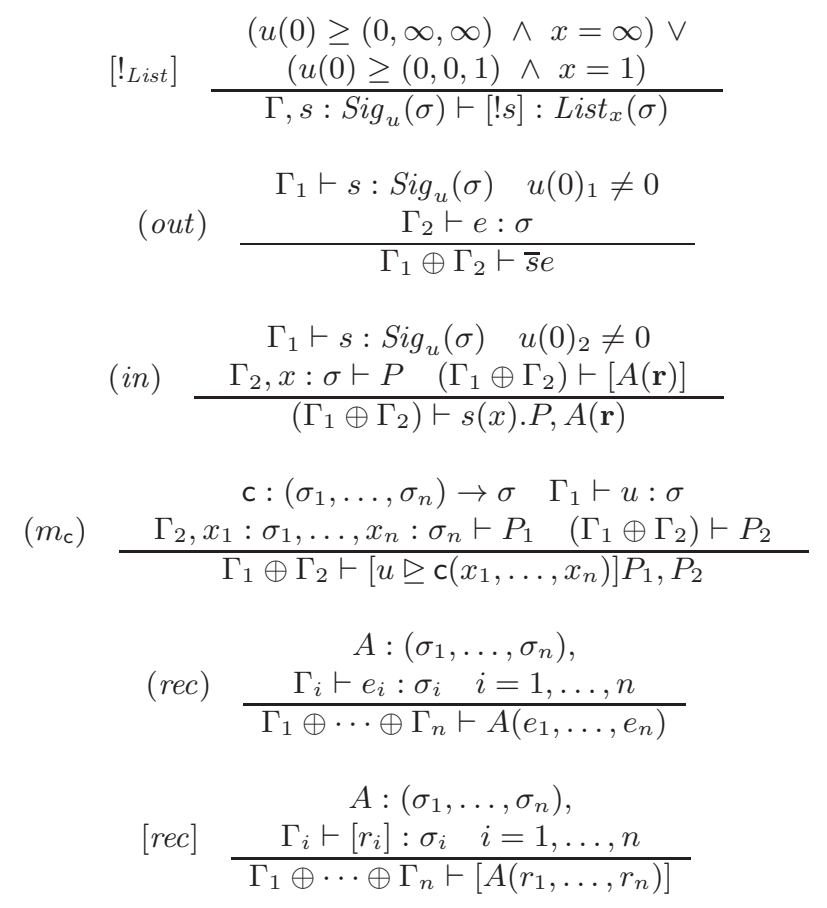

Table 4: Affine type system 
Finally, we turn to the typing of programs. We assume that each thread identifier $A$, defined by an equation $A\left(x_{1}, \ldots, x_{n}\right)=P$, comes with a type $\left(\sigma_{1}, \ldots, \sigma_{n}\right)$. Hence we require these types to be uniform. We also require that $A$ has the property that: (i) if $v_{i} \sim_{\sigma_{i}} u_{i}$ for $i=1, \ldots, n$ then $A\left(v_{1}, \ldots, v_{n}\right) \approx A\left(u_{1}, \ldots, u_{n}\right)$ and (ii) $x_{1}: \sigma_{1}, \ldots, x_{n}: \sigma_{n} \vdash P$ is derivable.

We also suppose that generated signals names are explicitly labelled with their types as in $\nu s: \rho P$. The labelled transition system in table 2 is adapted so that the output action carries the information on the types of the extruded names. This type is lifted by the rule (next) so that, e.g., $\nu s: \rho s .0, A(s) \stackrel{N}{\rightarrow} \nu s: \uparrow \rho A(s)$.

\section{Results}

We start by stating the expected weakening and substitution properties of the type system.

Lemma 6 (weakening) If $\Gamma \vdash U: T$ and $\left(\Gamma \oplus \Gamma^{\prime}\right) \downarrow$ then $\left(\Gamma \oplus \Gamma^{\prime}\right) \vdash U: T$.

Lemma 7 (substitution) If $\Gamma, x: \rho \vdash U: T, \Gamma^{\prime} \vdash v: \rho$, and $\left(\Gamma \oplus \Gamma^{\prime}\right) \downarrow$ then $\left(\Gamma \oplus \Gamma^{\prime}\right) \vdash$ $[v / x] U: T$.

Next we specify when a context $\Gamma$ is compatible with an action act, written $(\Gamma, a c t) \downarrow$. Recall that $V$ and $E$ denote a function from signals to finite lists of distinct values and finite sets of values, respectively. If $V(s)=\left[v_{1} ; \ldots ; v_{n}\right]$ then let $(V \backslash E)(s)=\left\{v_{1}, \ldots, v_{n}\right\} \backslash E(s)$. Then define a program $P_{(V \backslash E)}$ as the parallel composition of emissions $\bar{s} v$ such that $v \in$ $(V \backslash E)(s)$. Intuitively, this is the emission on an appropriate signal of all the values which are in $V$ but not in $E$. We also let $P_{V}$ stand for $P_{(V \backslash \emptyset)}$ where $\emptyset(s)=\emptyset$ for every signal $s$.

Definition 8 With each action act, we associate a minimal program $P_{\text {act }}$ that allows the action to take place:

$$
P_{a c t}= \begin{cases}0 & \text { if act }=\tau \text { or act }=N \\ \bar{s} v & \text { if act }=s v \text { or act }=s ? v \\ s(x) \cdot 0,0 & \text { if act }=\bar{s} v \\ P_{V \backslash E} & \text { if act }=(E, V)\end{cases}
$$

Definition 9 (compatibility context and action) $A$ context $\Gamma$ is compatible with an action act, written $(\Gamma$, act $) \downarrow$, if $\exists \Gamma^{\prime}\left(\Gamma \oplus \Gamma^{\prime}\right) \downarrow$ and $\Gamma^{\prime} \vdash P_{\text {act }}$.

We can now introduce the concept of typed transition which is a transition labelled with an action act of a program typable in a context $\Gamma$ such that $\Gamma$ and act are compatible.

Definition 10 (typed transition) We write $P \underset{\Gamma}{\stackrel{a c t}{\longrightarrow}} Q(P \underset{\Gamma}{\stackrel{\text { act }}{\Rightarrow}} Q)$ if: (1) $\Gamma \vdash P$, (2) $(\Gamma$, act $) \downarrow$, and (3) $P \stackrel{\text { act }}{\longrightarrow} Q(P \stackrel{\text { act }}{\Rightarrow} Q$, respectively).

Next, we introduce the notion of residual context which is intuitively the context left after a typed transition. (the definition for the auxiliary actions is available in appendix B). First, we notice that given a (uniform) type $\sigma$ and a value $v$ we can define as follows the minimum context $\Delta(v, \sigma)$ such that $\Delta(v, \sigma) \vdash v: \sigma$. Notice that $\Delta(v, \sigma)$ is the empty context if $f n(v)=\emptyset$ and it is a neutral context if $\sigma$ is non-affine. 


$$
\begin{array}{ll}
\Delta(s, \sigma) & =s: \sigma \\
\Delta\left(\mathrm{c}\left(v_{1}, \ldots, v_{n}\right)\right) & =\Delta\left(v_{1}, \sigma_{1}\right) \oplus \cdots \oplus \Delta\left(v_{n}, \sigma_{n}\right) \quad \text { if } c:\left(\sigma_{1}, \ldots, \sigma_{n}\right) \rightarrow \sigma
\end{array}
$$

Definition 11 (residual context) Given a context $\Gamma$ and a compatible and relevant action $\alpha$, the residual context $\Gamma(\alpha)$ is defined as follows:

$$
\Gamma(\alpha)= \begin{cases}\Gamma & \text { if } \alpha=\tau \\ \uparrow \Gamma & \text { if } \alpha=N \\ \left(\Gamma, \mathbf{t}: \sigma^{\prime}\right) \ominus \Delta\left(v: \sigma^{\prime}\right) \oplus\left\{s: \operatorname{Sig}_{u_{5}}\left(\sigma^{\prime}\right)\right\} & \text { if } \Gamma(s)=\operatorname{Sig}_{u}\left(\sigma^{\prime}\right), \alpha=\nu \mathbf{t}: \sigma^{\prime} \bar{s} v, \text { and }(1) \\ \Gamma \oplus \Delta\left(v, \sigma^{\prime}\right) \oplus\left\{s: \operatorname{Sig}_{u_{\text {out }}}\left(\sigma^{\prime}\right)\right\} & \text { if } \Gamma(s)=\operatorname{Sig}_{u}\left(\sigma^{\prime}\right), \alpha=s v, \text { and }(2)\end{cases}
$$

(1) $u_{5}=(0,1,0) \cdot(0,0,0)^{\omega}$ if $u \in U(5)$ and it is neutral otherwise (i.e., $\left.u \in U(2)\right)$.

(2) $u_{\text {out }}$ is the least usage of the same kind as $u$ which allows to perform an output within the instant (always defined).

The notion of residual context is instrumental to a precise statement of the way transitions affect the typing. First we notice that the type of expressions is preserved by the evaluation relation.

Lemma 12 (expression evaluation) If $\Gamma \vdash e: \rho$ and $e \Downarrow v$ then $\Gamma \vdash v: \rho$.

The following lemma records the effect of the substitution at the end of the instant.

Lemma 13 (substitution, end of instant) (1) If $\Gamma \vdash[A(\mathbf{r})], \Gamma^{\prime} \vdash P_{V}$, and $\left(\Gamma \oplus \Gamma^{\prime}\right) \downarrow$ then $\uparrow\left(\Gamma \oplus \Gamma^{\prime}\right) \vdash V(A(\mathbf{r}))$.

(2) If moreover there are $V^{\prime}, E$ such that $V, V^{\prime} \|-E$ then $V(A(\mathbf{r})) \approx V^{\prime}(A(\mathbf{r}))$.

Finally, the subject reduction theorem states that the residual of a typed transition is typable in the residual context (again, the residual context on auxiliary actions is defined in appendix B).

Theorem 14 (subject reduction) If $P \underset{\Gamma}{\stackrel{a c t}{\longrightarrow}} Q$ then $\Gamma($ act $) \vdash Q$.

Next we introduce a notion of typed bisimulation which refines the one given in definition 4 by focusing on typed processes and typed transitions. Let $C x t$ be the set of contexts and if $\Gamma \in C x t$ let $\operatorname{Pr}(\Gamma)$ be the set of programs typable in the context $\Gamma$.

Definition 15 (typed bisimulation) A typed bisimulation is a function $\mathcal{R}$ indexed on Cxt such that for every context $\Gamma, \mathcal{R}_{\Gamma}$ is a symmetric relation on $\operatorname{Pr}(\Gamma)$ such that: $P \mathcal{R}_{\Gamma} Q$, $P \underset{\Gamma}{\stackrel{\alpha}{\longrightarrow}} P^{\prime}$, bn $(\alpha) \cap f n(Q)=\emptyset$ implies $\exists Q^{\prime}\left(Q \underset{\Gamma}{\stackrel{\alpha}{\Rightarrow}} Q^{\prime}, \quad P^{\prime} \mathcal{R}_{\Gamma(\alpha)} Q^{\prime}\right)$. We denote with $\approx^{t}$ the largest typed labelled bisimulation.

An expected property of typed bisimulation is that it is a weaker property than untyped bisimulation: if we cannot distinguish two processes by doing arbitrary actions we cannot distinguish them when doing actions which are compatible with the typing. 
Proposition 16 If $P, Q \in \operatorname{Pr}(\Gamma)$ and $P \approx Q$ then $P \approx_{\Gamma}^{t} Q$.

We write $P \underset{\Gamma}{\stackrel{\tau}{\longrightarrow}} Q$ if $P \underset{\Gamma}{\stackrel{\tau}{\longrightarrow}} Q$ or $P=Q$. The following lemma states a strong commutation property of typed $\tau$ actions and it entails that typed bisimulation is invariant under $\tau$-actions.

Lemma 17 (1) If $P \underset{\Gamma}{\stackrel{\tau}{\longrightarrow}} P_{i}$ for $i=1,2$ then there is a $Q$ such $P_{i} \underset{\Gamma}{\stackrel{\tau}{\longrightarrow}} Q$ for $i=1,2$.

(2) If $P \underset{\Gamma}{\stackrel{\tau}{\Rightarrow}} Q$ then $P \approx_{\Gamma}^{t} Q$.

The second key property is that the computation at the end of the instant is deterministic.

Lemma 18 If $P \stackrel{N}{\Gamma} P_{i}$ for $i=1,2$ then $P_{1} \approx_{\uparrow(\Gamma)}^{t} P_{2}$.

Combining the two lemmas, we derive that typable programs are deterministic.

Theorem 19 (determinacy) If $P \underset{\Gamma}{\stackrel{N}{\Rightarrow}} \cdot \underset{\Gamma^{\prime}}{\stackrel{N}{\Rightarrow}} \cdots \underset{\Gamma^{\prime}}{\stackrel{N}{\Rightarrow}} P_{i}, i=1,2, \Gamma^{\prime}=\uparrow \Gamma$ then $P_{1} \approx_{\Gamma^{\prime}}^{t} P_{2}$.

Thus a typable process $P$ is deterministic in the following sense: whenever $C$ is a context such that $C[P]$ is typable and $C[P]$ reduces to $P_{1}, P_{2}$ after $n \geq 0$ instants, we can conclude that $P_{1}$ and $P_{2}$ are (typed) bisimilar. In other terms, as long as a typable process $P$ interacts with an environment $C$ according to the typing rules, at any instant, its behaviour is completely determined up to typed bisimulation.

\section{References}

[1] R. Amadio. The SL synchronous language, revisited. Journal of Logic and Algebraic Programming, 70:121-150, 2007.

[2] R. Amadio. A synchronous $\pi$-calculus. Information and Computation, 205(9):1470-1490, 2007.

[3] R. Amadio, I. Castellani and D. Sangiorgi. On bisimulations for the asynchronous $\pi$-calculus. In Theoretical Computer Science, 195:291-324, 1998.

[4] R. Amadio, M. Dogguy. Determinacy in a synchronous $\pi$-calculus. Technical Report, Université Paris 7 , Laboratoire PPS, July 2007. To appear in From semantics to computer science: essays in honor of Gilles Kahn, Y. Bertot et al (eds.), Cambridge University Press.

[5] G. Berry and G. Gonthier. The Esterel synchronous programming language. Science of computer programming, 19(2):87-152, 1992.

[6] F. Boussinot and R. De Simone. The SL synchronous language. IEEE Trans. on Software Engineering, 22(4):256-266, 1996.

[7] J.-Y. Girard. Linear Logic. Theoretical Computer Science,50(1):1-102, 1987.

[8] K. Honda and N. Yoshida. On reduction-based process semantics. Theoretical Computer Science, 151(2):437-486, 1995.

[9] N. Kobayashi. Type systems for concurrent programs. In Proc. 10th Anniversary Colloquium of UNU/IIST, Springer LNCS 2757, 2003.

[10] N. Kobayashi, B. Pierce, and D. Turner. Linearity and the pi-calculus. ACM Transactions on Programming Languages and Systems (TOPLAS), 21(5), 1999.

[11] L. Mandel and M. Pouzet. ReactiveML, a reactive extension to ML. In Proc. ACM Principles and Practice of Declarative Programming, pages 82-93, 2005.

[12] R. Milner. Communication and concurrency. Prentice-Hall, 1989.

[13] R. Milner, J. Parrow, and D. Walker. A calculus of mobile processes, parts 1-2. Information and Computation, 100(1):1-77, 1992.

[14] Ph. Wadler. A Taste of Linear Logic. In Proc. Mathematical Foundations of Computer Science, SLNCS 711, pages 185-210, 1993. 


\section{A Typing examples}

We type the programming examples introduced in section 1 .

Example 20 (typing the cell) With reference to example 1, assume an inductive (nonaffine) type State to represent the state of a cell and let $\sigma=\operatorname{Sig}_{u}($ State $)$ where $u=(\infty, 0, \infty)^{\omega}$ and $\sigma^{\prime}=\operatorname{List}_{\infty}(\sigma)$. Then we can require: Cell : $\left(\right.$ State, $\left.\sigma, \sigma^{\prime}\right)$ and Send: $\left(\right.$ State, $\left.\sigma, \sigma^{\prime}, \sigma^{\prime}\right)$. Because, the usage of the signals under consideration is $(\infty, 0, \infty)^{\omega}$, the type of their dereferenciation is $\operatorname{Set}_{\infty}\left(\right.$ State) and therefore we must require next $:\left(\right.$ State, $_{\text {Set }}($ State $\left.)\right) \rightarrow$ State, which means that the result of the function next must be invariant under permutations of the list of (distinct) states.

Example 21 (typing the synchronous data flow) With reference to example Q we assume an inductive type $D$ of data and let $\sigma=\operatorname{Sig}_{u}(D), \sigma_{I}=\operatorname{Sig}_{u_{I}}(D)$, and $\sigma_{O}=\operatorname{Sig}_{u_{O}}(D)$, where: $u=(1,1,0)^{\omega}, u_{I}=(0,1,0)^{\omega}$, and $u_{O}=(1,0,0)^{\omega}$. Then we can require: $A$ : $\left(\sigma_{I}, \sigma_{O}, \sigma_{I}, \sigma_{O}\right), B:\left(\sigma_{I}, \sigma_{O}, \sigma_{I}, \sigma_{O}\right)$, and $C:\left(\sigma_{I}, \sigma_{O}\right)$. The restricted signals $s_{2}, \ldots, s_{5}$ take the type $\sigma$ and the overall system is well-typed with respect to the context $s_{1}: \sigma_{I}, s_{6}: \sigma_{O}$.

Remark 22 (affinity vs. linearity) With reference to the data flow example 2, one may notice that the type system guarantees determinacy by making sure that at every instant at most one value is emitted on every signal. One could consider a more refined type system that guarantees that exactly one value is emitted on a signal at every instant 2 However, to obtain this system it is not enough to require that all linear hypotheses in the context are used in the typing. For instance, consider: $\nu s, s^{\prime}: \sigma\left(A\left(s, s^{\prime}\right) \mid A\left(s^{\prime}, s\right)\right)$ where: $\sigma=\operatorname{Sig}_{(1,1,0) \omega}, A:(\sigma, \sigma)$, and $A\left(s, s^{\prime}\right)=s() \cdot\left(\overline{s^{\prime}} \mid\right.$ pause. $\left.A\left(s, s^{\prime}\right)\right), A\left(s, s^{\prime}\right)$. This program could be linearly typed but it is stuck at every instant. Following previous work (see, e.g., [9]), one way to address this problem is to partition signals in a finite set of regions and to order them. Then one designs typing rules that require that a reception on a signal belonging to a given region only guards (prefixes) emissions on signals belonging to higher regions.

Example 23 (typing the client-server) With reference to example 3, assume an inductive (non-affine) type $D$ of data. Let $\sigma_{1}=\operatorname{Sig}_{u_{1}}(D)$ where $u_{1}=(1,0,0)^{\omega}$ be the type of the signals on which the server will eventually provide an answer. $\operatorname{Let}_{\operatorname{Req}}\left(\sigma_{1}, D\right)=$ req of $\sigma_{r}, D$ be the type of requests which are pairs composed of a signal and a datum. Let $\sigma_{\text {set }}=\operatorname{Set}_{1}\left(\operatorname{Req}_{1}\left(\sigma_{1}, D\right)\right)$ be the type of the set of requests issued by the clients. Let $\sigma=\operatorname{Sig}_{u}\left(\operatorname{Req}_{1}\left(\sigma_{1}, D\right)\right)$ with $u=(\infty, 0,1)^{\omega}$ be the type of the signal on which the server gets the requests and $\sigma^{\prime}=\operatorname{Sig}_{u^{\prime}}\left(\operatorname{Req}_{1}\left(\sigma_{1}, D\right)\right)$, with $u^{\prime}=(\infty, 0,0)^{\omega}$, the related type of the signal on which the clients send the requests. Finally, let $\sigma_{t}=\operatorname{Sig}_{u}(D)$ be the type of the signal on which the client sends the received answer (with a suitable usage $u$ ). Then we can type Server and Client as follows: Server: $(\sigma)$, Handle : $\left(\sigma, \sigma_{\text {set }}\right)$, and Client $:\left(D, \sigma^{\prime}, \sigma_{t}\right)$.

\section{B Residual context on auxiliary actions}

We specify the notion of residual context on auxiliary actions. The definition for the actions $s ? v$ is similar to the one for the actions $s v$. On the other hand, for the actions $(E, V)$, we

\footnotetext{
${ }^{2}$ In this system the 'else' branch of the input operator would become useless
} 
have to analyse how a program exports and imports usages at the end of the instant. For instance, consider $P=\overline{s_{1}} t_{1}\left|\overline{s_{2}} t_{2}\right| A\left(! s_{1}\right)$, and suppose $P \underset{\Gamma}{\stackrel{(E, V)}{\longrightarrow}} A\left(V\left(s_{1}\right)\right)$ where:

$$
E=\left[\left\{t_{1}\right\} / s_{1},\left\{t_{2}\right\} / s_{2}\right] \quad V=\left[\left[t_{1} ; t_{3}\right] / s_{1},\left[t_{4} ; t_{2}\right] / s_{2}\right] .
$$

The function $E$ represents what $P$ emits, the function $V$ represents what $P$ assumes to be emitted, moreover looking at the context $\Gamma$, we may determine what the process $P$ may receive at the end of the instant (note that $P$ may receive what it emits and that a value with an affine typing can be received at most once). In computing the residual context, we have to subtract what is exported to the environment while adding what is imported from it. Going back to our example, clearly the context $\Gamma$ must specify that $P$ may receive on $s_{1}$ at the end of the instant. Suppose moreover that it specifies that $P$ may not receive on $s_{2}$. Then in computing the residual context, we have to subtract the usage for $t_{2}$ which is exported to the environment while adding the usage for $t_{3}$ which is received from it. Following these considerations, we define:

$$
\begin{array}{lll}
\Delta(E, \Gamma)=\oplus\left\{\Delta(v, \lambda) \mid \Gamma(s)=\operatorname{Sig}_{u}(\lambda), v \in E(s), u(0)_{3} \neq 1\right\} & \text { (export) } \\
\Delta(V, \Gamma)=\oplus\left\{\Delta(v, \sigma) \mid \Gamma(s)=\operatorname{Sig}_{u}(\sigma), v \in V(s), u(0)_{3} \neq 0\right\} & \text { (import) }
\end{array}
$$

Note that in the 'exported context' $\Delta(E, \Gamma)$ we only care about usages of values of affine type, as otherwise $\Delta(v, \kappa)$ is neutral. On the other hand, in the 'imported context' we look at all the values regardless of their type. Indeed, $v$ might have a neutral type but contain a fresh signal name and then we need to import a neutral context to type it. Also note that in the following definition 24, we actually focus only on the values that are not emitted (in $E$ ).

Definition 24 (residual context on auxiliary actions) Given a context $\Gamma$ and an auxiliary action aux the residual context $\Gamma($ aux $)$ is defined as follows where $u_{5}$ is as in definition 11:

$$
\Gamma(\text { aux })= \begin{cases}\left(\Gamma \ominus\left\{s: \operatorname{Sig}_{u_{5}}\left(\sigma^{\prime}\right)\right\}\right) \oplus \Delta\left(v, \sigma^{\prime}\right) & \text { if } \Gamma(s)=\operatorname{Sig}_{u}\left(\sigma^{\prime}\right), \text { aux }=s ? v, \text { and }(1) \\ (\uparrow \Gamma \ominus \Delta(E, \Gamma)) \oplus \Delta\left(V^{\prime}, \Gamma\right) & \text { if aux }=(E, V) \text { and } V \backslash E=V^{\prime}\end{cases}
$$

